\title{
Intelligent Solutions for Screening Employees with Skills in High Demand
}

\author{
Sylvia Encheva ${ }^{1 *}$ \\ ${ }^{1}$ Stord/Haugesund University College, Bjørnsonsg. 45, 5528 Haugesund, Norway \\ ${ }^{*}$ Corresponding author
}

\begin{abstract}
Today's project leaders face situations where workforce optimizations have to be considered not only under a project planning period but also a number of times through the entire project period. Such occurrences require efficient ways of selecting minimum number of employees with skills that are called for. A lot of efforts and manual labor can be spared by involvement of smart assistive technology. Executing workforce related search utilized by specific graphs and defeasible logic is proposed in this work.
\end{abstract}

Keywords- Kneser graphs; service; management

\section{INTRODUCTION}

Current developments in world's economy require optimizations in all organization related processes. "When forming a team, the ideal is to balance the skills of one team member with the complementary abilities of others", [9]. Unnecessary overlapping of skills and expertise in projects is what very few can afford if at all. "Given the high costs associated with field labor, optimizing worker efficiency and productivity has become an absolute imperative", [10]. Modern IT based applications provide a large number of options for assisting establishments in their attempts to ameliorate management of workforce. A wearable interactive tool suitable for instant picturing of relations between employees and their skills can considerably speed up the process of workforce composition and distribution. An implement of this kind can also support automation of advanced levels of work-flow management. In this work we propose application of various graphs for modeling such a tool supported by defeasible reasoning. The latter is chosen due to its ability to provide efficient reasoning in the presence of incomplete, inconsistent and conflicting information in addition to modeling of business rules and policies, where rules with exceptions are often used, [3].

The rest of the paper is organized as follows. Related work and supporting theory may be found in Section 2. The obtained results are presented in Section 3. The paper ends with a conclusion in Section 4.

\section{RELATED WORK}

Graph related statements are taken mainly from [4] and [5]. The Petersen graph has ten vertices and fifteen edges. It appeared first in 1886 in a paper written by A. B. Kempe but carries the name of Julius Petersen presenting it in 1898. The Petersen family contains seven graphs that can be constructed from the Petersen graph. The Clebsch graph is strongly related to the Petersen graph. Any vertex of the Clebsch graph has ten non-neighbors bringing up another Petersen graph each.

Martin Kneser was the first one to discover essential features of Kneser graphs, [6]. A vertex in a Kneser graph $\mathrm{K}(\mathrm{n}, \mathrm{k})$ is a $k$-element subset of a set with cardinality $n$. Any vertex is adjacent to $\left(\begin{array}{c}n-k \\ k\end{array}\right)$ vertices where any two adjacent vertices stand for two disjoint sets. A bipartite Kneser graph $\mathrm{H}(\mathrm{n}, \mathrm{k})$ has two types of vertices representing $k$ and $n-k$ elements belonging to a set with cardinality $n$. Any two adjacent vertices satisfy the condition that one of them is a subset of the other.

An Odd graph is a Kneser graph with $\left(\begin{array}{c}2 n-1 \\ n-1\end{array}\right)$ vertices and $\frac{n\left(\begin{array}{c}2 n-1 \\ n-1\end{array}\right)}{2}$ edges. A vertex of the Odd graph can be seen as a subset with cardinality $n-1$ of a set with cardinality $2 n-1$.

Defeasible reasoning is a rule-based approach to reasoning with incomplete and inconsistent information, [1]. The main advantage of this approach is the combination of two desirable features: enhanced representational capabilities allowing one to reason with incomplete and contradictory information, coupled with low computational complexity compared to mainstream nonmonotonic reasoning, [3].

Defeasible logic presented here is as in [2]. A defeasible theory (a knowledge base in defeasible logic, or a defeasible logic program) consists of five different kinds of knowledge: facts, strict rules, defeasible rules, defeaters, and a superiority relation. Facts are indisputable statements. Strict rules are rules in the classical sense: whenever the premises are indisputable (e.g. facts) then so is the conclusion.

Defeaters are rules that cannot be used to draw any conclusions. Their only use is to prevent some conclusions. In other words, they are used to defeat some defeasible rules by producing evidence to the contrary. The superiority relation among rules is used to define priorities among rules, that is, where one rule may override the conclusion of another rule. Notice that a cycle in the superiority relation is counterintuitive. Another point worth noting is that, in defeasible logic, priorities are local in the following sense: Two rules are considered to be competing with one another only if they have 
complementary heads. Thus, since the superiority relation is used to resolve conflicts among competing rules, it is only used to compare rules with complementary heads; the information $r_{1}>r_{2}$ for rules $r_{1}, r_{2}$ without complementary heads may be part of the superiority relation, but has no effect on the proof theory.

Defeasible logic has attracted significant attention in nonmonotonic reasoning and found many applications, particularly in expert systems and knowledge-based systems communities, [7].

Outcomes of proof theory allow representation by a Venn diagrams.

\section{EMPLOYEES AND THEIR SKILLS}

Below we focus on versatile approaches for selecting minimum number of personnel with requested expertise and consider cases where complementary skills are of special interest.

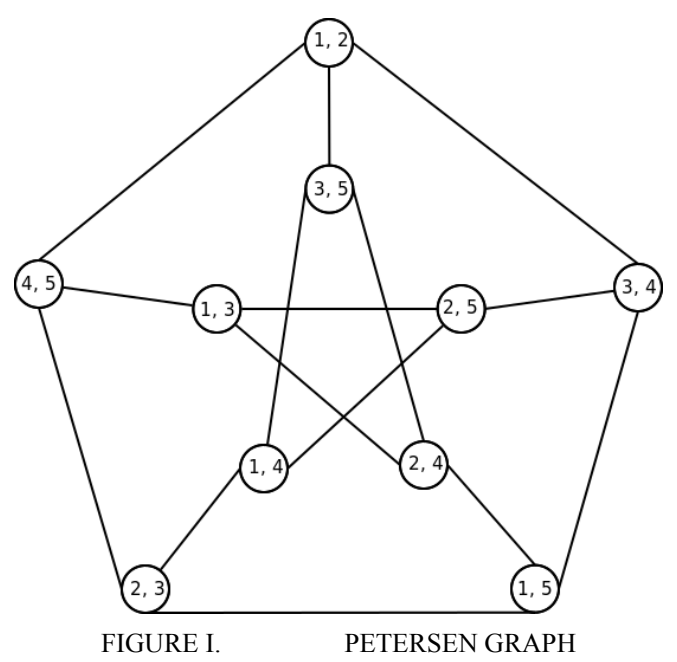

Interpretation of the Petersen graph as a Kneser graph is shown in [8]. Petersen graph shown in Fig. 1 can be used in the following way. Suppose there are ten people possessing five skills. A vertex represents a person described with two skills and two vertices are connected by an edge if the corresponding two people do not share a skill, i.e. they posses complimentary skills. Thus any three vertices where one of them is connected to the other two with exactly two edges provide a quick selection of three people possessing all the five skills that the team covers.

Another very useful interpretation of this graph is that there is a person possessing any two of the five skills. In case a job requires two of the five skills a project leader can choose to assign two or three different people or just one to complete that job, depending on the amount of work and deadlines. In that sence two people will be needed if one of the skills has to be exercised by more than one person while three people will be required in case both skills have to be exercised by more than one person.
The Odd graph in Fig. 2 can be used for a case of seven skills and 35 people where each person is described by three skills and two vertices are connected if they refer to two people with complementary skills. Vertices are placed on four circles where the most inner circle contains fourteen vertices and the other three circles contain seven vertices each. Each vertex from the most inner circle (an example is colled in red) is adjacent to one of the vertices in any of the four circles. All vertices situated on the three most outer circles are adjacent to two of the verteces on most inner circle and to two of the verteces on the same circle where they are placed. Examples are collered in blue, yellow and green, respectively. This allows application of the Odd graph also in case people are given different roles, responsibilities and authorizations. Note that cases involving authorizations have to be carefully examined for eventual appearance of defeasible rules.

Cases with complementary skills and shared skills are discussed further down.

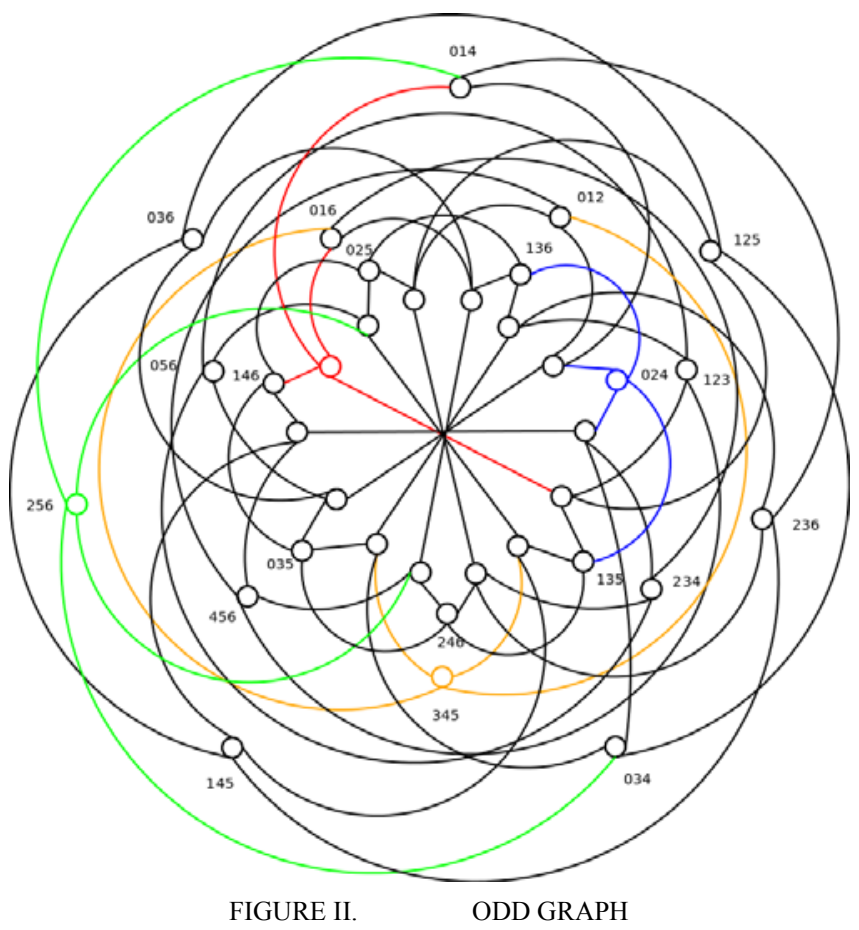

Suppose three skills $(1,3,4)$ are needed, Fig. 3. Three people possessing two of the skills are described as $(1,4),(1,3)$, $(3,4)$. If all the skills are needed a person with complimentary skills $(2,5)$ should join the team. Such arrangements can be quite useful if all or some of the skills $(1,3,4)$ require two people to complete the job while the job requiring skills $(2,5)$ can be completed by a single person. 


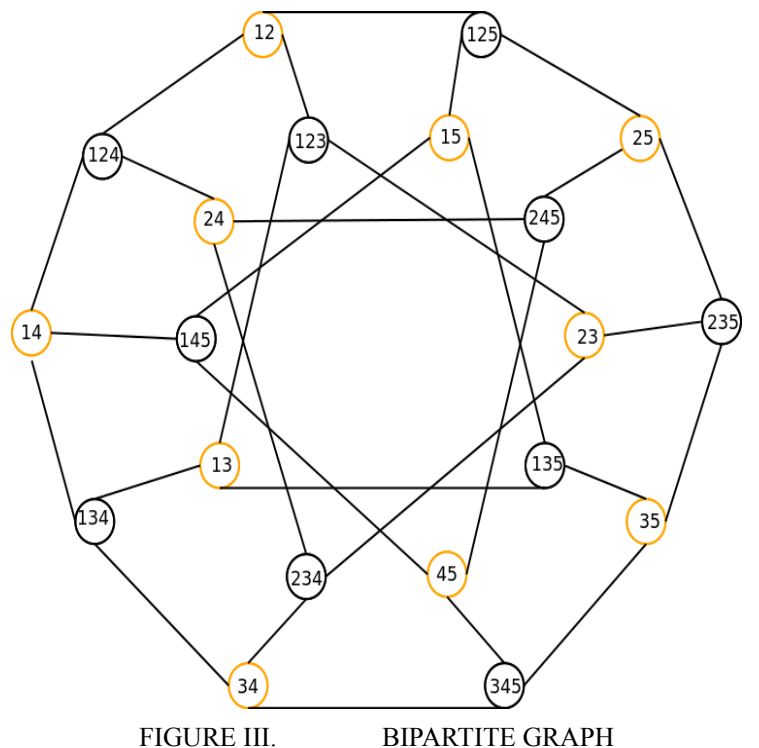

A possibility for assembling two teams with complementary skills is presented in Fig. 4 (it is a part of Petersen graph family). The first team is 'TL, E1, E3, E5' and the second one is 'TL, E2, E4, E6' and 'TL' is a team leader for both. In this case every employee from one of the teams shares a skill with any of the employees from the other team.

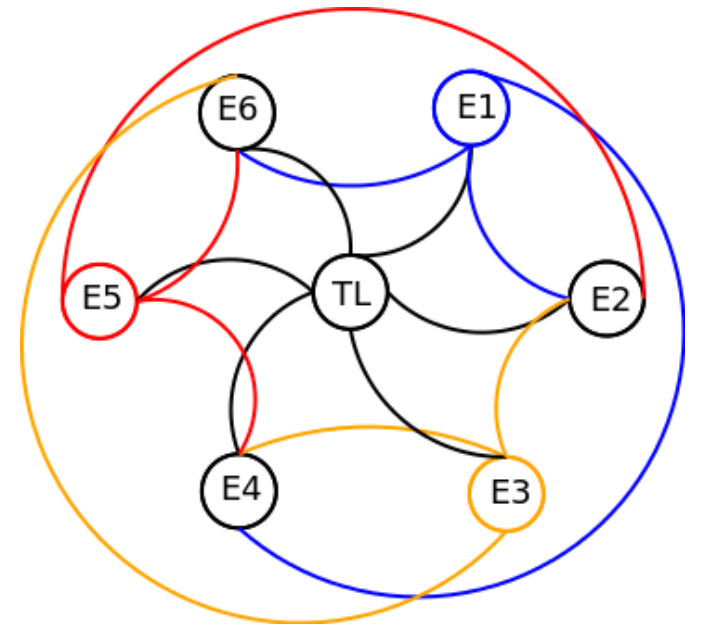

FIGURE IV.

A GRAPH BEING PART OF PETERSEN FAMILY

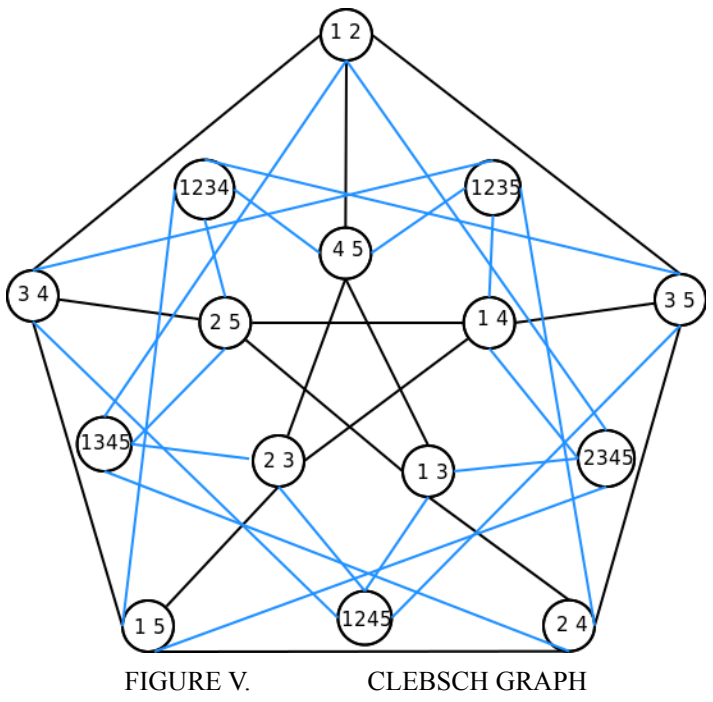

The Clebsch graph in Fig. 5 can be used to describe a team of fifteen people possessing five skills. Five people posses four skills each where any of them is connected with 4 other people characterized by two skills such that one of the two skills is possessed by the person with four skills. Vertices representing people characterized by two skills are connected when their skills are complementary.

Incorporating defeasible reasoning in the process of recommending a particular employee can increase the likelihood of a successful outcome.

\section{CONCLUSION}

The approach suggested in this article is suitable for being used by mobile devices. Another advantage of using such visualization is that one can see all participants and their respective skills, while a search in a database will provide information connected to that particular search. Being able to see all the whole picture might significantly effect the final choice and it can definitely envisage future solutions. Such a tool can support automation of advanced levels of work-flow management.

\section{REFERENCES}

[1] G. Antoniou, N. Dimaresis, G. Governatori, "A modal and deontic defeasible reasoning system for modelling policies and multi-agent systems," Expert Systems with Applications, vol. 36, pp. 4125-4134, 2009 .

[2] G. Antoniou, D. Billington, G. Governatori, and M. J. Maher, "Representation results for defeasible logic," Journal ACM Transactions on Computational Logic (TOCL) TOCL Homepage archive vol. 2 (2), pp. 255-287, 2001.

[3] N. Bassiliades, G. Antoniou, I. Vlahavas, "A Defeasible Logic Reasoner for the Semantic Web," LNCS 3323, pp. 49-64, 2004.

[4] N. L. Biggs, Algebraic Graph Theory, 2nd ed. Cambridge, England: Cambridge University Press, 1993.

[5] B. Bollobas, Extremal Graph Theory, Dover, 2004.

[6] M. Kneser, "Aufgabe 360", Jahresbericht der Deutschen MathematikerVereinigung, 2. Abteilung 58: 27., 1955.

[7] M. Rohaninezhad, S. M. Arif, and S. A. M. Noah, "A grounder for SPINdle defeasible logic reasoner", Expert Systems with Applications, 42, pp. 7098-7109, 2015. 
[8] http://math.stackexchange.com/questions/269326/petersen-graphprolems

[9] http://smallbusiness.chron.com/complementary-skills-team-81389.html

[10] https://www.accenture.com/hu-en/insight-enabling-digital-workforcetransmission-distribution.aspx 